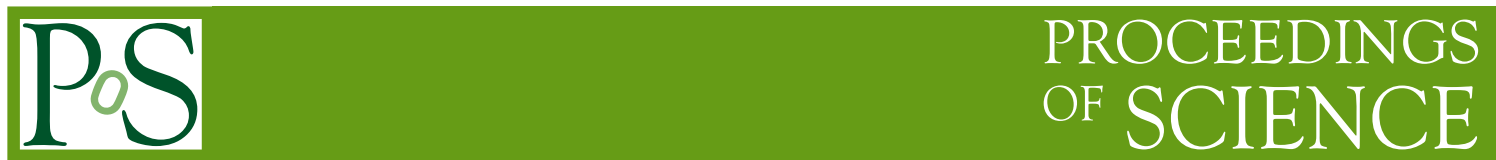

\title{
Investigation into polarization uncertainty minimization of solid polarized targets
}

\section{Dustin Keller*i}

University of Virginia

E-mail: dustinejlab.org

\begin{abstract}
A discussion of uncertainty minimization in the polarization produced by Dynamic Nuclear Polarization is presented. Techniques of error estimation and minimization are outlined for the use in polarized data analysis. Procedures for minimizing uncertainty in a setting such as the Jefferson Lab solid polarized target experiments are described with examples using recent experiments. Both proton and deuteron targets are considered. Techniques in the deuteron tensor polarization enhancement and enhanced tensor polarization measurement uncertainty are also discussed in the interest and preparation of future experiments.
\end{abstract}

XVth International Workshop on Polarized Sources, Targets, and Polarimetry,

September 9-13, 2013

Charlottesville, Virginia, USA

\footnotetext{
${ }^{*}$ Speaker.

${ }^{\dagger}$ A footnote may follow.
} 


\section{Introduction}

The uncertainty in Nuclear Magnetic Resonance (NMR) measurements of polarization in a solid polarized target can frequently produce the largest contribution to the systematic uncertainty of the polarized observables. The $12 \mathrm{GeV}$ upgrade at Jefferson Lab seeds a new generation of polarized target experiments which continue to probe over a larger kinematic range and to greater precision. A detailed understanding and the minimization of the polarization errors is now more critical than ever.

The uncertainty in the calibration of the polarization measurement primarily come from measurement limitation of area of the NMR signal which can greatly depend on the noise level in the signal. The second leading contribution is the error in temperature of the target material at thermal equilibrium which is determined by measurement accuracy and degree of fluctuations. Other instrumental and environment factors may also play a role in the overall error.

The solid polarized target experiments for use in an electron beam use targets as either the deuteron using $\mathrm{ND}_{3}$ or the proton using $\mathrm{NH}_{3}$. There have been multiple experiments using these targets with an evaporation fridge such as E93-026, E01-006, E08-027, E07-003, and E06-014 at Jefferson Lab and E143, E155, and E155x at SLAC.

Future Jefferson Lab experiments for which polarization uncertainty minimization is relevant using a solid polarized target include the experiments E12-07-107, E12-09-009, E12-06-109, and E12-09-007 using the Hall B horizontal fridge, as well as the experiment E12-11-108 using SOLID in Hall A, and the tensor asymmetry experiment E12-13-011 in Hall C. The latter experiment requires a solid tensor polarized target.

Some of the essential aspects of the polarization systematic uncertainty for the proton and the deuteron are discussed as well as the measurement capacity of the deuteron spin alignment. For a more detailed outline of the integrated systematic uncertainty and minimization procedure refer to [1].

\section{Polarized Target Data Analysis}

Dynamic Nuclear Polarization (DNP) of the solid material used in nuclear experiments can be achieved at $\sim 1 \mathrm{~K}$ using a homogeneous magnetic holding field and a microwave field to transfer the spin polarization from the electrons to the nuclei. The solid polarized target system, see Figure 1 and Figure 6 , uses a ${ }^{4} \mathrm{He}$ evaporation refrigerator with sufficiently high cooling power to minimize the heating effects of the microwaves and high beam current. A superconducting magnet is used to polarize and maintain polarization of the target material during the experiment.

\subsection{The NMR System}

The nuclear spin polarization is measured with a continuous-wave NMR coil and Liverpool Q-meter [2]. The Q-meter works as part of a circuit with phase sensitivity designed to respond to the change of the impedance in the NMR coil. The radio frequent (RF) susceptibility of the material is inductively coupled to the NMR coil which is part of a series LCR circuit, tuned to the Larmor frequency of the nuclei being probed. The output, consisting of a DC level subtracted by a post-Q-meter conditioning card (Yale card), is digitized and recorded as a target event [B]]. 


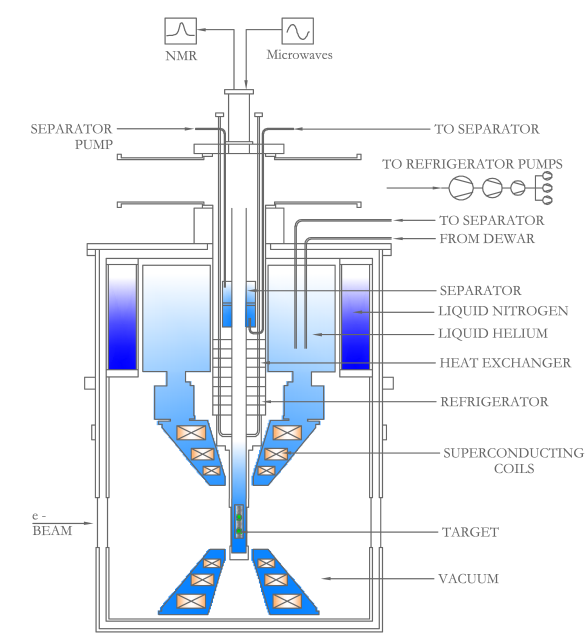

Figure 1: Polarized target system for $\mathrm{NH}_{3} / \mathrm{ND}_{3}$ at $5 \mathrm{~T}$ used to achieve a temperature of $\sim 1 \mathrm{~K}$ (Diagram by A. Rijal).

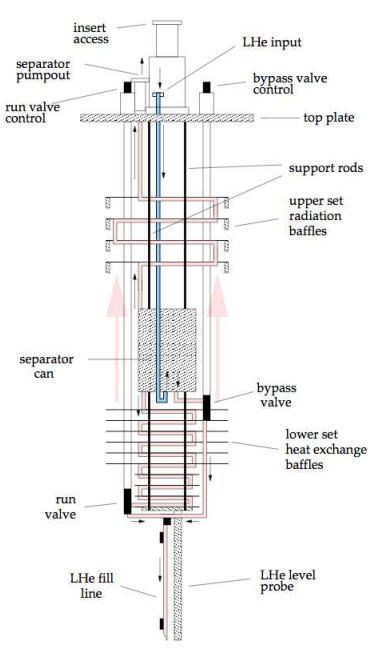

Figure 2: The fridge of the target system.

A target NMR calibration measurement or Thermal Equilibrium measurement (TE) is used to find a proportionality constant to determine the enhanced polarization under a range of thermal conditions given the area of the "Q-curve" NMR signal at the same magnetic field. The magnetic moment in the external field results in a set of $2 J+1$ energy sublevels through Zeeman interaction, where $J$ is the particle spin. The TE natural polarization for a spin-1/2 particle is given by,

$$
P_{T E}=\tanh \left(\frac{\mu B}{k T}\right)
$$

where $\mu$ is the magnetic moment in the external field of strength $B, k$ is the Boltzmann constant, and $T$ the temperature. Measuring $P_{T E}$ at low temperature increases stability and the polarization signal. This is favorable being that the uncertainty in the NMR signal increases as the area of the signal decreases. The uncertainty due to temperature in $P_{T E}$ for a spin-1/2 target can be expressed as,

$$
\delta P_{T E}=\delta T \frac{\mu B}{K T^{2}} \operatorname{sech}^{2}\left(\frac{\mu B}{K T}\right) .
$$

The dynamic polarization value is derived by comparing the enhanced signal $S_{E}$ integrated over the driving frequency $\omega$ leading to $P_{E}=G C_{T E} A_{E}$ with calibration constant defined as $C_{T E}=$ $P_{T E} / A_{T E}$, and $G$ being the gain from the Yale conditioning card, where $P_{E}\left(A_{E}\right)$ is the polarization (area) of the enhanced signal and $P_{T E}\left(A_{T E}\right)$ is the polarization (area) from the thermal equilibrium measurement. The uncertainty in target calibration from the thermal equilibrium measurement is only dependent on the error in the TE polarization due to temperature $\delta P_{T E}$ and the error in the area of the TE signal $\delta A_{T E}$ when the error in $B$ is negligible.

\subsection{Instrumental Contributions}

There is some uncertainty that involves polarization variation due to instrumental limitations 


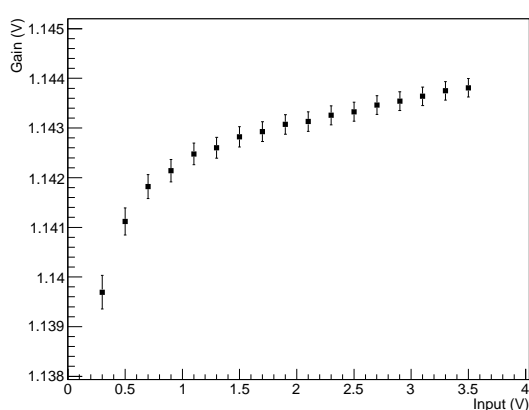

Figure 3: Yale card gains as a function of positive input voltage.

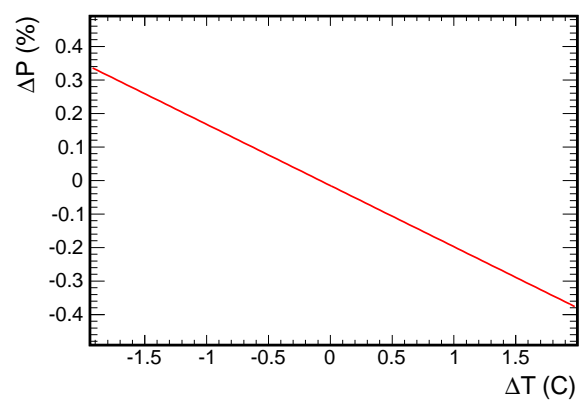

Figure 4: Polarization deviation trend as a function of change in Q-meter temperature.

or influences from the environment. A parameterization of this variation can be used to reduce or at least set a range in the polarization uncertainty. As an example consider the characterization curve, Figure 3, of the Yale gain card. Understanding the gain as a function of input voltage and the range of input voltage over a segment of target data events enables one to know the variation in polarization over that segment.

As an additional example it is possible to quantify the expected variation in polarization based on the change in temperature of the Q-meter. The Q-meter circuit sensitivity to ambient temperature can result in deviations in the integrated signal generating additional error in the polarization. The modulator output signal has a small temperature dependence that can be seen with constant input voltage and phase difference. The deviation in polarization as a function of the change in temperature is shown in Figure 9 .

In both the examples only an error in polarization can be acquired based on the operating range of the dependent variable. However, making the dependent variable part of the target data stream allows for a correction to the polarization based on the functional dependence, reducing the error estimate.

\subsection{NMR Signal Fitting and Integration}

The "Q-curve" baseline spectrum response must be measured in order to subtract it from the recorded signal data, see Figure 5. After baseline subtraction the remnant background is fitted with a second degree polynomial fit which is then subtracted to give the final TE spectrum, integrated with a Riemann sum to acquire the area. In order to obtain an error estimate in the area a known signal lineshape and area can be generated on a standard baseline. The area is then measured using the described technique and compared to the known generated area. A simple Gaussian can be used for the spin-1/2 but for deuterons a distinct lineshape is used [4]. It is also possible to simulate the noise in the signal by studying the signal to noise ratio recorded during a TE measurement and generate narrow Gaussian spikes of various amplitude throughout the simulated TE singnal. There is a much larger error in area if the signal is not situated in the center of the baseline so that after baseline subtraction the signal sits on a line with a large slope. The polynomial subtraction can then somewhat distort the area increasing the resulting error. Figure 6 shows the signal after 


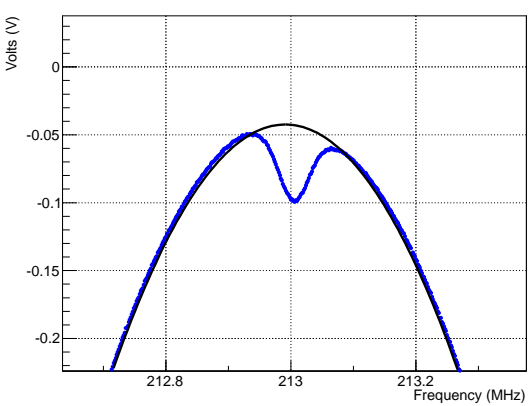

Figure 5: A proton TE signal before baseline subtraction.

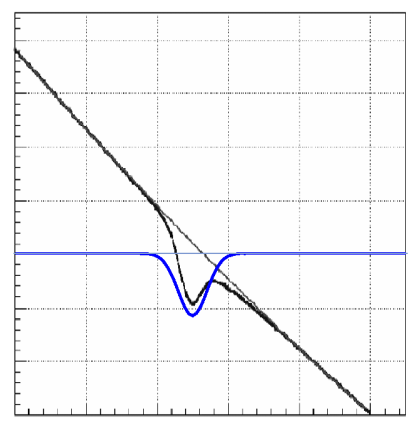

Figure 6: Different types of polynomial background fits.

background subtraction of a signal that was in the center and one that was significantly off-center. Studies at UVA indicate the uncertainty in TE area for the proton can be as large as 2\% [1] and for the deuteron $2.6 \%$. With care to center the signal and with the use of a high quality baseline the error can drop to less than $1 \%$ for the proton and $1.5 \%$ for the deuteron. The deuteron noise to signal ratio is higher due the smaller size of the TE signal. Deuteron noise level can be address with NMR improvements and alteration such as the cold NMR [5].

\section{Usability Criteria}

Accurate error bars on the integrated area points enables fitting of the decay constant $t_{1}$ to establish the time requirement for thermal equilibrium. After thermalization has been achieved a series of systematic steps are followed to extract the maximum amount of information with minimal uncertainty. The degree of thermal fluctuation at true TE should not exceed $2 \%$ in area at $1 \mathrm{~K}$ over 20 minutes. To ensure good TE data some quality constraints are set on area and pressure using a linear hypothesis.

The full range of the TE area and pressure measurements is analyzed selecting the data that leads to the smallest slope over the largest set of points. A quality constraint is set such that at least six points in area (pressure) spanning the time range in which a fit to a two parameter line returns a slope less than a fixed limit. The slope limit comes from an allowance of $2 \%$ change from point to point in TE area under a one parameter line hypothesis over six points. The number of contiguous points in the final one parameter line fit are increased until the two parameter line fit slope condition is no longer met or the uncertainty in the one parameter line fit increases with the addition rather than decreases. The one parameter line fit is then used to obtain the final area and pressure and associated uncertainties in these values.

\section{Uncertainty in Tensor Polarization}

The deuteron, a spin-1 target, has energy levels which split three ways in a magnetic field $m=-1,0,+1$. The target spin orientation can be described using the vector and tensor polarization. Tensor polarization can be expressed in terms of the vector polarization as $P_{z z} \doteq 2-\sqrt{4-3 P^{2}}$ 


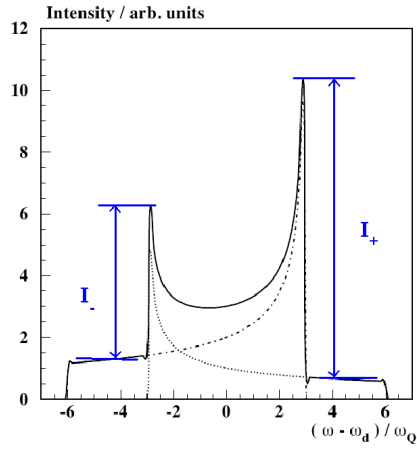

Figure 7: Deuteron Magnetic Resonance line shape and peak intensities $I_{+}$and $I_{-}$.

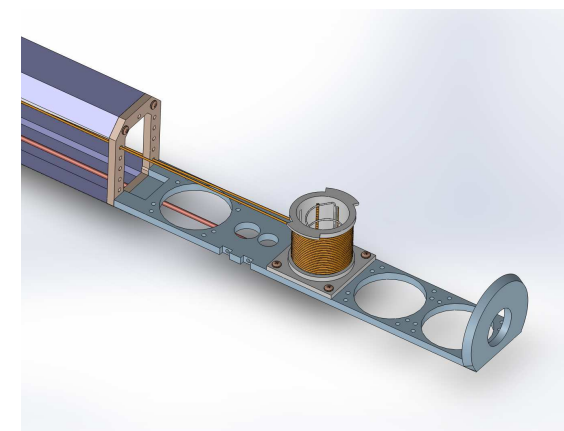

Figure 8: Target cup and coil used to generate the modulated RF field (Diagram by A. Rijal).

where the dot implies the relation is true when thermal equilibrium exists within the deuteron spin species. The definition of vector polarization for spin-1 is,

$$
P=\frac{n_{+}-n_{-}}{n_{+}+n_{-}+n_{0}} \doteq \frac{r^{2}-1}{r^{2}+r+1},
$$

with the tensor polarization defined as,

$$
P_{z z}=\frac{n_{+}-2 n_{0}+n_{-}}{n_{+}+n_{-}+n_{0}} \doteq \frac{r^{2}-2 r+1}{r^{2}+r+1} .
$$

Where the second equality in each case is not part of the definition but a relation to the transition ratio $r$ defined as $r=I_{+} / I_{-}$, see Figure 7 . These relations in terms of $r=e^{\beta \hbar \omega_{d}}$ are true to first order in $\beta \hbar \omega_{q}$, where $\omega_{d}\left(\omega_{q}\right)$ is Larmor (quadrapole interaction) frequency. The intensities $I_{-}$and $I_{+}$can be determined using a fitting procedure [4]. The resulting polarization measurements can be used complementary to the TE signal polarization calibration. For vector polarizations over $30 \%$ the two independent methods result in a discrepancy smaller than $2.5 \%$. This leads to a minimum uncertainty in the natural tensor polarization of approximately $5 \%$.

\section{Enhanced Tensor Polarization}

Deuteron spin alignment can be manipulated when exposed to a modulated RF field using an external coil around the target cup, as seen if Figure 8. The accuracy and enhancement of the tensor polarization is greatly dependent on the polarization technique. For the electron beam the focus is presently on positive enhanced tensor polarization which can be expressed as,

$$
P_{z z}=\frac{\left|\left(I_{+}-I_{-}\right) P\right|}{I_{+}+I_{-}}-\delta p_{a}
$$

Here $P$ is the vector polarization that was achieved before the RF-modulation. A positive tensor polarization enhancement occurs only when the $n_{+}+n_{-}$population increases with respect to $n_{0}$ population. To optimize the tensor polarization the vector polarization must be maximized using DNP at which point the microwave is turned off and the RF-modulation begins. The RF-modulation 
induces thermal cooling in the $n_{0}$ sublevel effectively suppressing transitions between either the $m=-1 \rightarrow m=0$ or $m=0 \rightarrow m+=1$. There is a correction $\delta p_{a}$ which is the reduction to the polarization due to the overlap of the transition frequencies between -3 and 3 in Figure 7. The reduction can be estimated by determining the ratio between the overlap area to the overall area under the DMR line. An additional correction could be made to take into account the change in polarization seen in the reduction in the overall area due to the spin relaxation. Full saturation occurs when the area under a single transition peak and corresponding pedestal has been reduced to zero. Tensor optimization will occur when the range in RF-modulation is chosen as to maximize the area of the resulting signal while minimizing the area in the overlap. Due to the propagation in error from the measurement of $I_{+}$and $I_{-}$as well as the error in area from integrating using a Riemann sum the minimal projected uncertainty in positive tensor polarization is $9-12 \%$ using this RF technique.

A solid tensor polarized target using both negative and positive tensor polarizations is plausible for use with a photon beam or low current electron beam. One way to achieve negative tensor polarization is to use dynamic nuclear orientation on a material that contains a separate proton spin-spin reservoir such as the -diol compounds. Applying a RF-modulation slightly off the Larmor frequency of the proton spins can lead to enhanced alignment of the deuteron spins independent of the vector polarization. The off-resonance RF field cools the proton spin-spin interaction reservoir and is transmitted to the deuteron quadrupole interaction reservoir [6]. Negative enhanced tensor polarization can be expressed as,

$$
P_{z z}=C\left|I_{+}-I_{-}\right|
$$

Where $C$ is a calibration constant achieved from a TE measurement. In this expression a negative calibration constant is assumed with either orientation $I_{+}\left(I_{-}\right)$or $I_{-}\left(I_{+}\right)$being below (above) the x-axis leading to negative values of $P_{z z}$. Because the deuteron spin alignment increases in a natural distribution there is no distortion in the DMR line from the RF-modulation as seen when the modulating is around the deuteron Larmor frequency. This can result in much more accurate representation of the tensor polarization. The minimal projected uncertainty using this technique is $4-6 \%$. The majority of uncertainty here is from the TE calibration as previously outlined.

\section{Conclusion}

An investigation is made to understand and reduce possible systematic errors in both the deuteron and proton targets. The largest errors arises from the thermal equilibrium calibration measurements emerging from the uncertainty in signal area and the temperature measurements. Additional quality constrains can be imposed to ensure that only quality TE data is used. For the deuteron tensor polarization the measuring ability and associated uncertainty is also considered. Only a projection of the uncertainties based on technique are given at this time. Data confirmation

will come in future studies. Optimization of tensor polarization occurs by maximizing the deuteron spin alignment for which the degree of accuracy of the polarization must be well understood.

\section{References}

[1] D. Keller, Nucl. Instr. and Meth. A728:133 (2013) 
[2] G.R. Court, et al. Nucl. Instr. and Meth. A324:433 (1993)

[3] D. Crabb, W. Meyer Solid Polarized Targets for Nuclear and Particle Physics Experiments Nucl. Part. Sci. 47:67-109 (1997)

[4] C. Dulya, et al. Nucl. Instr. and Meth. in Phys. Res. A 398, 109-125(1997)

[5] G.R. Court, et al. Nucl. Instr. and Meth. in Phys. Res. A 527, 253 (2004)

[6] de Boer, W. et al. Phys.Lett. B46 143-144 (1973) 